\title{
Mechanobiology in harness
}

\author{
Understanding how cells sense and adapt to their environment, and engineering defined culture \\ substrates, will be central to progress in tissue engineering and regenerative medicine.
}

Cells are active gels. By remodelling their cytoskeleton, they deform and migrate. In fact, because the cytoskeleton is usually coupled to the extracellular matrix by focal adhesions, cells can pull on the matrix not only to generate the traction forces needed for migration, but also to sense its mechanical properties and structure. And in addition to mechanical signals, cells can receive biochemical cues from small molecules, proteins or matrix-degradation by-products dissolved in the matrix or attached to it.

Both biochemical and mechanical signals - which are transduced and regulated by the intracellular network of protein interactions - are used by the cell to alter its behaviour: for example, to change shape, migrate, reprogram itself into another cell and, in the case of a stem cell, differentiate into a more specific cell type.

A few of the mechanosensing, mechanotransduction and intracellularsignalling processes involved in cell behaviour are fairly well understood (for example, the activation of stress-sensitive transmembrane proteins such as ion channels or integrins, or the alignment of endothelial cells induced by fluid shear flow), and some are beginning to be controlled (such as the effects, on stem cell differentiation, of matrix stiffness and topographical order at the nanoscale). However, being able to exploit the mechanisms of cellular mechanobiology for biomedical purposes remains a dream.

Yet as Dave Dingal and Dennis Discher conclude in a Commentary on page 532, "with the wide range of material and analytical technologies emerging to help the translation from in vitro to in vivo, challenges in concepts and applications seem increasingly addressable with suitable rigour and reproducibility". The authors highlight the importance of both physical and chemical factors in stem cell differentiation and self-renewal by providing examples of synergetic effects arising from soluble factors and culture substrates (from their mechanical properties or their bound factors). And as Morgan Alexander, Chris Denning and colleagues assert in a Review article on page 570 , culture substrates that can be scaled up to produce the billions of cells needed to meet the future demands of regenerative medicine and pharmaceutical drug screening need to be fully synthetic, and the culture conditions fully defined. They also
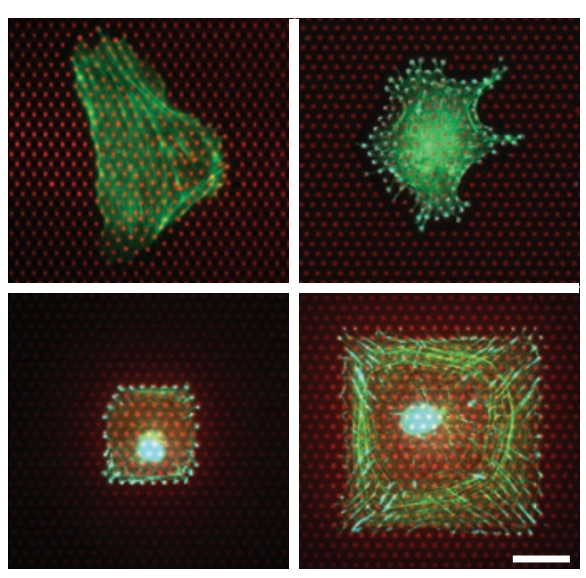

Live cells on microposts of varying stiffnesses. Reproduced from Yang, M. T. et al. Nature Protoc. 6, 187-213 (2011). Scale bar, $20 \mu \mathrm{m}$.

argue that both experimental high-throughput materials discovery and modelling methods that enable structure-property relationships will play a prime role in the search and discovery of new substrates for cell expansion.

There would be less need for highthroughput materials discovery if we had a firm grasp of how the coupling of matrix mechanics and biochemical factors, and their interplay with cellular signalling, directs stem cell fate. As William Murphy, Todd McDevitt and Adam Engler state in a Review article on page 547, "the understanding of how distinct material characteristics combine to regulate stem cell fate is still in its infancy". In fact, there exist only a handful of examples of material-induced stem cell fate in chemically defined cell culture environments. To be sure, synthesis strategies that have enabled the systematic exploration of the effects of the chemistry, structure and mechanical properties of materials on cell behaviour have only been mastered in the last several years. For example, understanding how surface texture at the nanoscale is transduced by interactions with the cells' integrin receptors - discussed by Matthew Dalby, Nikolaj Gadegaard and Richard Oreffo in a Review article on page 558 - requires recent advances in precision nanoscale patterning (for example, nanolithography, etching or anodizing).

We also include in this focus issue on cell culture four developments that, as a whole, span mechanistic, phenomenological, methodological and engineering aspects of the understanding and application of cellular mechanobiology. On page 631, Pere Roca Cusachs, Xavier Trepat and colleagues show with myoepithelial cells that cells can sense matrix stiffness through variations in the dynamics of integrinmatrix bonds. In a linked News \& Views article (page 539), José García and Andrés García add that these results highlight the importance of targeting specific integrins in therapies aimed to treat tissue dysfunction. Kristi Anseth and collaborators report on page 645 that stem cell fate can also be influenced by past mechanical environments, and that such 'mechanical memory' is mediated by two transcriptional co-activators known to be involved in critical regulatory signalling pathways. Although it is unclear how mechanical information is retained by the cell - as Jeroen Eyckmans and Christopher Chen mention in an accompanying News \& Views article (page 542) - cell culture materials that allow for the dynamical control of substrate mechanics are poised to become consequential.

Even more puzzling is the observation, reported by Kevin Chalut and co-authors on page 638, of embryonic stem cells in transition towards differentiation for which their nucleus behaves as an auxetic material - that is, when stretched it expands in the transversal direction, contrary to the behaviour of common materials. Ning Wang discusses potential explanations for the phenomenon in an associated News \& Views (page 540).

Cell culture is routinely performed on glass or plastic, which hardly resemble the cells' natural milieu. Soft substrates can be a more favourable environment, as Jianping Fu and colleagues show for the case of the production of functional motor neurons derived from human pluripotent stem cells (page 599). In a linked News \& Views article (page 543), Emily Lowry and Christopher Henderson wonder whether the efficacy of the soft substrates on cell yield can also be modulated by soluble factors. Likewise, mechanical cues from synthetic materials can influence, and may be able to mimic, the effects of potent soluble factors. The fate of mechanobiology, however, lies on synergistically harnessing both. 